\title{
FATORES ASSOCIADOS À VITALIDADE AO NASCER
}

\author{
Viviane Prado ${ }^{1}$ \\ Kátia Biagio Fontes ${ }^{2}$ \\ Kayna Trombini Schmidt ${ }^{3}$
}

PRADO, V.; FONTES, K. B.; SCHMIDT, K. T. Fatores associados a vitalidade ao nascer. Arq. Cienc. Saúde UNIPAR, Umuarama, v. 19, n. 1, p, 39-40, jan./abr. 2015.

RESUMO: O objetivo deste estudo foi identificar os fatores associados à vitalidade do recém-nascido no município de Cianorte, Paraná. Realizou-se estudo descritivo e transversal, no qual foram utilizados dados das 1519 Declarações de Nascidos Vivos de 2011. Foi considerada como variável resposta a vitalidade do recém-nascido, avaliada por meio do índice de apgar de 1' e 5'. Na avaliação da associação entre variáveis, empregou-se o teste de qui-quadrado de Pearson e o teste exato de Fisher. Conforme os dados obtidos, os fatores envolvidos na vitalidade do recém-nascido, foram: estado civil e município de moradia maternos, número de consultas de pré-natal, sexo, raça, idade gestacional e peso do recém-nascido. A realização deste estudo possibilitou identificar fatores associados à vitalidade do recém-nascido e, sobretudo, trazer à tona a reflexão sobre o modelo assistencial designado ao atendimento das gestantes e as práticas obstétricas.

PALAVRAS-CHAVE: Nascimento vivo; Índice de Apgar; Saúde Materno-Infantil.

\section{FACTORS ASSOCIATED WITH VITALITY AT BIRTH}

\begin{abstract}
This study aims to identify factors related to the vitality of newborns in the city of Cianorte, Paraná, through a transversal descriptive study using data from 1,519 Statement of Live Births in 2011. The variable used was the positive response related to the newborn, evaluated through 1' and 5' apgar score. The Pearson Chi-square test and the Fisher exact test were applied in order to evaluate the association between variables. According to data obtained, the factors regarding the vitality of newborns through 1' and 5' apgar score were: mother's marital status and city of residence, number of prenatal consultations, sex, race, gestational age and weight of the newborn. Through this study, it was possible to identify factors associated to the vitality of newborns and, above all, to bring up some reflection on the care model concerning pregnant patients and obstetric practices.
\end{abstract}

KEYWORDS: Live birth; Apgar score; Maternal and Child Health.

\section{Introdução}

Atualmente, a mortalidade neonatal (entre 0 e 27 dias de vida) é responsável por quase $70 \%$ das mortes no primeiro ano de vida. O número elevado de mortes por asfixia intraparto, sobretudo de crianças com peso adequado ao nascer e em gravidez de baixo risco, demonstra o grande potencial de evitabilidade dessas mortes. No Brasil, nascem cerca de três milhões de crianças ao ano, das quais $98 \%$ em hospitais. Sabe-se que a maioria delas nasce com boa vitalidade, entretanto, manobras de reanimação podem ser necessárias de maneira inesperada (BRASIL, 2011).

Considera-se que o RN apresente boa vitalidade, quando ao nascimento, este é considerado a termo, está respirando ou chorando, com tônus muscular em flexão, sem a presença de líquido amniótico meconial, não necessitando consequentemente de qualquer manobra de reanimação. Porém, caso o RN necessite de reanimação, a utilização da escala de Apgar permite avaliar a resposta do RN às manobras de reanimação realizadas e a eficácia destas (BRASIL, 2008). A avaliação pela escala deve ser feita no $1^{\circ}$ e $5^{\circ} \mathrm{mi}-$ nutos de vida, devendo ser repetida a cada cinco minutos, nos casos em que o escore permanecer baixo, até atingir o valor acima de seis (VIEGAS; VILHENA-MORAES, 1996). Dessa forma, os valores registrados na escala de Apgar no $1^{\circ}$ e $5^{\circ}$ minutos são importantes indicadores das condições de nascimento (BRASIL, 2008).

Resultados de estudos anteriores têm demonstrado que um índice de Apgar baixo ao nascer tem se mostrado um fator condicionante de morte neonatal. Estudo epidemiológico que analisou os casos de óbito neonatal ocorridos demonstrou que a maioria deles apresentou baixo Apgar (ROCHA e tal., 2011). Outros estudos nacionais que analisaram a associação entre o escore de Apgar e mortalidade neonatal corroboram com esta informação ao apresentarem associação estatisticamente significativa (NASCIMENTO et al., 2012; OLIVEIRA et al., 2012; LANSKY, 2014), sendo que resultado de Apgar menor que sete apresentou-se como fator de risco para mortalidade neonatal em neonatos com baixo peso ao nascer (ALMEIDA et al., 2011; GAIVA; FUJIMORI; SATO, 2014).

Sendo assim, conhecer os fatores associados ao baixo Apgar, são de suma importância, pois servirão de subsídio para que sejam empreendidos esforços por parte dos serviços de saúde a populações que apresentem estas características.

A Declaração de Nascido-Vivo (DN) constitui um instrumento fundamental a fim de analisar os fatores envolvidos na vitalidade do $\mathrm{RN}$ ao nascer, pois permite a coleta de dados sobre características maternas referentes aos dados socioeconômicos, histórico da gestação pregressa e atual, além de registrar informações sobre o RN. Neste sentido, o objetivo deste estudo foi levantar os dados contidos nas declarações dos nascidos-vivos em 2011, no município de Cianorte - PR, e identificar os fatores associados à vitalidade do $\mathrm{RN}$ ao nascer por meio dos resultados da escala de Apgar no 1' e 5'. 


\section{Material e Método}

Trata-se de um estudo descritivo e transversal, de abordagem quantitativa. A amostra do estudo foi composta pela totalidade das DNs dos nascidos vivos no município de Cianorte, no período de janeiro a dezembro de 2011. Foram coletadas as informações referentes aos 1551 nascidos vivos nesse período, entretanto, fez-se necessário excluir 32 sujeitos em decorrência do preenchimento incompleto dos dados, totalizando 1519 DNs analisadas. O município onde foi realizada a pesquisa é referência para atendimento às gestantes de onze municípios da região, dos quais, cinco não oferecem assistência ao parto.

Todos os dados registrados nas DNs foram coletados durante o mês de junho de 2012. Os dados foram inseridos num banco de dados do Sotware Statistica 8.0 e realizada estatística descritiva. Para as comparações univariadas, utilizaram-se o teste qui-quadrado de Pearson e o teste exato de Fisher no Software R, tendo como variável dependente a vitalidade do $\mathrm{RN}$ avaliada por meio dos resultados dos escores de Apgar nos 1' e 5'. As DNs foram divididas em dois grupos: os neonatos que apresentaram escore no boletim de Apgar abaixo de sete e os iguais ou superior a sete. Com relação as variáveis independentes, foram consideradas a idade materna, estado civil, escolaridade, paridade anterior, tipo de gestação, tipo de parto, história de aborto, número de consultas pré-natal, idade gestacional, sexo, raça/cor e peso ao nascer. Foram consideradas significativas aquelas variáveis que atingiram a significância de $\mathrm{p}<0,05$.

$\mathrm{O}$ projeto foi submetido à apreciação do Comitê de Ética em pesquisa da Universidade Paranaense (CAAE: 02168812.7.0000.0109), sendo aprovado sob o parecer $n^{\circ}$ $13791 / 2012$. Vale salientar que foram respeitados todos os preceitos éticos, conforme Resolução 466/2012 do Conselho Nacional de Saúde.

\section{Resultados}

Das 1519 DNs analisadas observou-se que 35 $(2,3 \%)$ dos neonatos apresentaram baixo Apgar ao nascer no 1 'e no 2' este número reduziu para $9(0,6 \%)$. Foram registrados $13(0,85 \%)$ casos de má formação e $12(0,8 \%)$ de óbito.

Quando analisadas as características sociodemográficas maternas, pode-se verificar que a maioria eram casadas ou se encontravam em união estável, tinham entre 20 e 34 anos, estudaram oito anos ou mais e residiam em Cianorte. Somente as variáveis Estado civil, Apgar no 5' e Município de moradia no 1' apresentaram associação estatisticamente significativa. Quando analisada a proporção de RNs que apresentaram baixo Apgar no 1' e no 5' por local de moradia, a maioria das mães que relataram morar em outra localidade, apresentaram percentual mais elevado comparado as que relataram morar em Cianorte (Tabela 1).

Tabela 1: Análise univariada segundo Apgar no 1'e 5' em relação as variáveis relativas às características sociodemográficas maternas. Cianorte, PR, Brasil, 2011

\begin{tabular}{|c|c|c|c|c|c|c|c|}
\hline \multirow[t]{2}{*}{ Variável } & \multicolumn{2}{|c|}{ Apgar 1' } & \multirow[t]{2}{*}{ p-valor } & \multicolumn{2}{|c|}{ Apgar 5, } & \multirow[t]{2}{*}{$\%$} & \multirow[t]{2}{*}{ p-valor } \\
\hline & $<7$ & $\geq 7$ & & $<7$ & $\geq 7$ & & \\
\hline \multicolumn{8}{|l|}{ Estado civil } \\
\hline Solteiras & 9 & 184 & \multirow{3}{*}{0,0527} & 4 & 189 & 12,71 & \multirow{3}{*}{$0,0345 *$} \\
\hline Casadas/União estável & 26 & 1276 & & 5 & 1297 & 85,71 & \\
\hline Outros & 0 & 24 & & 0 & 24 & 1,58 & \\
\hline \multicolumn{8}{|l|}{ Faixa etária (anos) } \\
\hline$<21$ & 12 & 375 & \multirow{3}{*}{0,4456} & 2 & 385 & 25,48 & \multirow{3}{*}{$1 *$} \\
\hline $21-35$ & 20 & 994 & & 7 & 1007 & 66,75 & \\
\hline$\geq 35$ & 3 & 115 & & 0 & 118 & 7,77 & \\
\hline \multicolumn{8}{|l|}{ Escolaridade (anos) } \\
\hline$<4$ & 18 & 854 & \multirow[t]{3}{*}{0,7125} & 7 & 865 & 57,41 & \multirow[t]{3}{*}{$0,3365^{*}$} \\
\hline $4-7$ & 0 & 5 & & 0 & 5 & 0,33 & \\
\hline$\geq 8$ & 17 & 625 & & 2 & 640 & 42,26 & \\
\hline \multicolumn{8}{|l|}{ Município de moradia } \\
\hline Cianorte & 12 & 863 & \multirow{2}{*}{0,0047} & 4 & 871 & 57,6 & \multirow{2}{*}{$0,5067 *$} \\
\hline Outros & 23 & 621 & & 5 & 639 & 42,4 & \\
\hline
\end{tabular}

* Utilizado o teste exato de Fisher.

Em relação as características obstétricas pregressa materna predominaram as puérperas com única paridade anterior, sem histórico de aborto. Já quando analisada a gestação atual a maioria apresentou gestação única. Prevaleceram as puérperas com mais de quatro consultas no pré-natal e houve um percentual elevado de mulheres que realizaram mais de sete consultas pré-natais $(88,7 \%)$. Nesta análise a única variável que apresentou associação com Apgar foi nú- mero de consultas de pré-natal (Tabela 2). 
Tabela 2: Análise univariada segundo Apgar no 1'e 5' em relação as variáveis relativas às características maternas obstétricas pregressa, gestacional atual e de assistência pré-natal. Cianorte, PR, Brasil, 2011.

\begin{tabular}{|c|c|c|c|c|c|c|c|}
\hline \multirow[t]{2}{*}{ Variável } & \multicolumn{2}{|c|}{ Apgar 1' } & \multirow[t]{2}{*}{ p-valor } & \multicolumn{2}{|c|}{ Apgar 5, } & \multirow[t]{2}{*}{$\%$} & \multirow[t]{2}{*}{ p-valor } \\
\hline & $<7$ & $\geq 7$ & & $<7$ & $\geq 7$ & & \\
\hline \multicolumn{8}{|l|}{ Paridade anterior } \\
\hline$\geq 2$ & 5 & 336 & \multirow{2}{*}{0,2415} & 2 & 339 & 22,45 & \multirow{2}{*}{$1 *$} \\
\hline$<2$ & 30 & 1148 & & 7 & 1171 & $\mathbf{7 7 , 5 5}$ & \\
\hline \multicolumn{8}{|l|}{ Aborto } \\
\hline Sim & 1 & 8 & \multirow{2}{*}{$0,1897^{*}$} & 0 & 9 & 0,59 & \multirow{2}{*}{$1 *$} \\
\hline Não & 34 & 1476 & & 9 & 1501 & 99,41 & \\
\hline \multicolumn{8}{|l|}{ Tipo de gestação } \\
\hline Única & 33 & 1459 & \multirow[t]{2}{*}{$0,1265^{*}$} & 9 & 1483 & 98,22 & \multirow[t]{2}{*}{$1 *$} \\
\hline Múltipla & 2 & 25 & & 0 & 27 & 1,78 & \\
\hline \multicolumn{8}{|l|}{$\mathbf{N}^{0}$ de consultas $\mathrm{PN}$} \\
\hline$<4$ & 3 & 25 & \multirow{3}{*}{$0,0332 *$} & 0 & 28 & 1,84 & \\
\hline$\geq 4$ & 32 & 1456 & & 9 & 1479 & 97,96 & \\
\hline Ignorado & 0 & 3 & & 0 & 3 & 0,2 & \\
\hline
\end{tabular}

Constatou-se que todos RNs nasceram no ambiente hospitalar, a maioria era do sexo feminino, de raça branca, parto cesáreo, com idade gestacional $\geq 37$ semanas e com peso $\geq 2500 \mathrm{~g}$. As variáveis relacionadas ao $\mathrm{RN}$ que apresentaram associação estatisticamente significativa em relação ao Apgar no 1' foram sexo, raça, peso e idade gestacional. Apenas a tipo de parto não apresentou associação. Já quando comparadas as variáveis estudadas com Apgar 5', verificou-se que apenas raça, peso idade gestacional apresentaram associação. Quando analisada a proporção de casos de baixo Apgar'1 entre os sexos, observou-se que o sexo masculino apresentou maior percentual de casos $(3,3 \%)$ comparado ao feminino $(1,3 \%)$. Também houve uma maior proporção de RNs de raça não branca, com idade gestacional menor que 37 semanas e com peso menor que 2500 gramas que apresentaram maior proporção de casos de baixo Apgar no 1' e 5' minutos. Quando realizada a análise univariada constatou-se que apenas a variável tipo de parto não apresentou associação com Apgar no 1' e 5'. A raça, idade gestacional e peso do RN apresentaram associação com Apgar no 1' e 5'. Já a variável sexo apresentou associação apenas no 1' (Tabela 3).

Tabela 3: Análise univariada segundo Apgar no 1'e 5' em relação variáveis relativas ao sexo do RN, ao parto e atenção neonatal. Cianorte/PR, 2011

\begin{tabular}{|c|c|c|c|c|c|c|c|}
\hline \multirow[t]{2}{*}{ Variável } & \multicolumn{2}{|c|}{ Apgar 1' } & \multirow[t]{2}{*}{ p-valor } & \multicolumn{2}{|c|}{ Apgar 5, } & \multirow{2}{*}{$\%$} & \multirow{2}{*}{ p-valor } \\
\hline & $<7$ & $\geq 7$ & & $<7$ & $\geq 7$ & & \\
\hline \multicolumn{8}{|l|}{ Sexo } \\
\hline Masculino & 25 & 725 & \multirow{2}{*}{$\mathbf{0 , 0 0 8 3}$} & 7 & 743 & 49,37 & \multirow{2}{*}{$0,1043 *$} \\
\hline Feminino & 10 & 759 & & 2 & 767 & 50,63 & \\
\hline \multicolumn{8}{|l|}{ Raça } \\
\hline Branca & 28 & 1360 & \multirow{2}{*}{0,0153} & 6 & 1382 & 91,38 & \multirow{2}{*}{0,0358 * } \\
\hline Demais & 7 & 124 & & 3 & 128 & 8,62 & \\
\hline \multicolumn{8}{|l|}{ Idade gestacional } \\
\hline$<37$ semanas & 10 & 90 & \multirow{2}{*}{$<0,0001$} & 3 & 97 & 6,58 & \multirow{2}{*}{0,0174 * } \\
\hline$\geq 37$ semanas & 25 & 1394 & & 6 & 1413 & 93,42 & \\
\hline \multicolumn{8}{|l|}{ Tipo de parto } \\
\hline Vaginal & 17 & 671 & \multirow{2}{*}{0,6934} & 5 & 683 & 45,29 & \multirow{2}{*}{$0,7394 *$} \\
\hline Cesáreo & 18 & 813 & & 4 & 827 & 54,71 & \\
\hline \multicolumn{8}{|l|}{ Peso } \\
\hline$<2500 \mathrm{~g}$ & 11 & 95 & \multirow{2}{*}{$<0,0001$} & 3 & 103 & 6,98 & \multirow{2}{*}{0,0204 * } \\
\hline$\geq 2500 \mathrm{~g}$ & 24 & 1389 & & 6 & 1407 & 93,02 & \\
\hline
\end{tabular}

* Utilizado o teste exato de Fisher. 


\section{Discussão}

Foram registrados 1551 nascimentos por ocorrência, no município de Cianorte, em 2011. Ao comparar os dados de 2010, disponíveis online, aos registros analisados nesta pesquisa, houve um crescimento de $12 \%$ no total de nascidos vivos por ocorrência. A porcentagem de RNs prematuros foi de $6,6 \%$ e se assemelhou à média brasileira, que registrou 7,1\% de partos prematuros em 2010 (BRASIL, 2015).

Quando analisado as condições sociodemográficas maternas, o baixo Apgar esteve associado ao estado civil e local de moradia. Pesquisa anterior também evidenciou o estado civil como fator associado a baixa vitalidade (KILSZTAJN et al., 2007). O tempo gasto entre a casa e hospital maior que 60 minutos tem se confirmado como fator de risco para óbito neonatal (OR=2,31; IC95\%: 1,17-4,60) (NASCIMENTO et al., 2012). Estudo realizado no Brasil demonstrou que as maiores taxas de mortalidade neonatal ocorreram entre crianças cujas mães relataram peregrinação para obter assistência hospitalar ao parto, sugerindo lacunas na organização da rede de saúde (LANSKY et al., 2014).

Neste estudo nenhuma das variáveis relativas às características maternas obstétricas pregressas apresentou associação. O número de consultas no pré-natal foi a única variável relacionada a assistência pré-natal que apresentou associação com baixo Apgar, confirmando resultado de estudo anterior (KILSZTAJN et al., 2007). O número de consultas tem sido apontado em vários estudos como fator associado a mortalidade neonatal (RIBEIRO et al., 2009; SOARES; MENEZES, 2010), sendo demonstrado que o número insuficiente de consultas constitui-se como fator de risco para a mortalidade neonatal (NASCIMENTO et al., 2012; KASSAR et al., 2013; GAIVA; FUJIMORI; SATO, 2014). Em relação às consultas pré-natais, o MS preconiza que o número de avaliações pré-natais realizadas deve ser de no mínimo seis consultas (BRASIL, 2005). No estado do Paraná, em 2009, constatou-se um índice elevado de mulheres que realizaram mais do que sete consultas $(78,7 \%)$, entretanto, quando avaliados dados referentes ao Brasil, essa porcentagem diminuiu para 58,5\% (BRASIL, 2015). No presente estudo, $88,7 \%$ das mulheres realizaram mais que sete consultas pré-natais, indicando que as gestantes foram acompanhadas conforme o recomendado pelo MS.

De acordo com o Ministério da Saúde (MS), o parto normal é aconselhável e mais seguro em relação ao cesáreo, uma vez que diminui risco de complicações como hemorragia pós-parto, infecções puerperais e prematuridade (BRASIL, 2006). Os resultados deste estudo revelaram que a porcentagem de parto cesáreo foi de $57,5 \%$, corroborando com dados referentes ao Brasil, em 2009, com 50,1\% dos partos por cesárea (BRASIL, 2015). A incidência de partos cesáreos, no município de Cianorte e no Brasil, está acima do recomendado pela Organização Mundial de Saúde (OMS), que seria no mínimo de $15 \%$ com margem para porcentagem máxima de 25\% (OMS, 1996).

Apesar de outros estudos apresentarem associação estatisticamente significativa entre tipo de parto e mortalidade neonatal (RIBEIRO et al., 2009; SOARES; MENEZES, 2010), a variável parto cesáreo e baixa vitalidade ao nascer parece não estar associada ao baixo Apgar, pois neste estudo esta variável não apresentou associação corroborando com resultado de estudo anterior (KILSZTAJN et al., 2007). Outro estudo demonstrou que o parto cesáreo não resultou em fator de risco ou de proteção para baixo Apgar (SALVO, 2007). Contrariando este resultado, outro estudo evidenciou que a diminuição da vitalidade e parto cesáreo apresentaram associação (FRANCESCHINI; CUNHA, 2007).

Já as variáveis, sexo do bebê, raça, peso ao nascer, tipo de parto e idade gestacional demonstraram associação com baixo Apgar. Outro estudo aponta a associação entre sexo do RN e óbito neonatal (RIBEIRO et al., 2009). RNs do sexo masculino nascidos por cesárea tem apresentado associação com menor vitalidade (FRANCESCHINI; CUNHA, 2007). Quanto a raça, estudos anteriores tem apontado esta variável como fator de risco para mortalidade neonatal (CARVALHO et al., 2007; MAIA; SOUZA; MENDES, 2012) tendo maior razão de chance a raça preta/parda $(\mathrm{OR}=9,7)(\mathrm{CARVALHO}$ et al., 2007).

Estudos também indicara o baixo peso como fator de risco para baixo Apgar (SALVO et al., 2007) e a associação entre peso do RN e baixa vitalidade (KILSZTAJN et al., 2007) corroborando com resultado do presente estudo. O baixo peso aos nascer têm se confirmado como importante fator de risco para mortalidade neonatal (SOARES; MENEZES, 2010; GONÇALVES; COSTA; BRAGA, 2011; KASSAR et al., 2013; LANSKY et al., 2014).

Estudos têm evidenciado a associação entre idade gestacional e baixo Apgar (KILSZTAJN et al., 2007; FISHER; CARABALLO; SÁNCHEZ, 2011) confirmando resultado encontrado neste estudo. Alem disso estudos também têm evidenciado que RNs com idade gestacional inferior a 37 semanas têm maior chance de mortalidade neonatal quando comparados com RNs a termo (NASCIMENTO et al., 2012; GAIVA; FUJIMORI; SATO, 2014).

\section{Conclusão}

A realização deste estudo possibilitou identificar fatores associados à vitalidade do $\mathrm{RN}$, sendo encontradas associações estatisticamente significativas entre as variáveis relacionadas as características sociodemográficas maternas: estado civil e município de moradia; relacionada a gestação: idade gestacional; relacionados ao RN: sexo, raça e peso ao nascer; e relacionada a assistência no pré-natal, número de consultas de pré-natal.

A realização deste trabalho contribuiu, sobretudo, como meio de subsídio a fim de identificar populações vulneráveis e trazer à tona a reflexão sobre o modelo assistencial designado ao atendimento das gestantes e as práticas obstétricas no município e região adstrita em questão.

Entre as limitações encontradas no desenvolver da pesquisa, identificou-se a falta de registro de morbidades na DN como um impeditivo para a avaliação das causas de parto cesáreo.

\section{Referências}

ALMEIDA et al. Sobrevida e fatores de risco para mortalidade neonatal em uma coorte de nascidos vivos de muito baixo peso ao nascer, na Região Sul do Município de São Paulo, Brasil. Cad. Saúde Pública, v. 27, n.6, p. 10881098, 2011. 
BRASIL. Agência Nacional de Saúde Suplementar. O modelo de atenção obstétrica no setor de Saúde Suplementar no Brasil: cenários e perspectivas. Rio de Janeiro: ANS, 2008.158 p. Disponível em: < http://bvsms. saude.gov.br/bvs/publicacoes/modelo_atencao_obstetrica. pdf>. Acesso em: 01 ab. 2015.

BRASIL. Ministério da Saúde. Secretaria de Atenção à Saúde. Departamento de Ações Programáticas Estratégicas. Área Técnica de Saúde da Mulher. Pré-natal e puerpério: atenção qualificada e humanizada. Brasília: Ministério da Saúde, 2005. 158p. Disponível em: http://bvsms.saude. gov.br/bvs/publicacoes/pre-natal_puerperio_atencao_ humanizada.pdf Acesso em: 06 abr. 2015.

BRASIL. Ministério da Saúde. Secretaria de Atenção à Saúde. Departamento de Ações Programáticas e Estratégicas. Atenção à saúde do recém-nascido: guia para os profissionais de saúde. Ministério da Saúde, Secretaria de Atenção à Saúde, Departamento de Ações Programáticas e Estratégicas. Brasília: Ministério da Saúde, 2011. 195p. Disponível em: <http://bvsms.saude. gov.br/bvs/publicacoes/atencao_recem_nascido_\%20guia_ profissionais_saude_v1.pdf $>$. Acesso em: 01 ab. 2015.

BRASIL. Ministério da Saúde. DATASUS: Informações em Saúde. Morbidade Hospitalar do SUS. Disponível em: $<$ www.datasus.gov.br/tabnet/tabnet.htm $>$. Acesso em: 01 ab. 2015 .

BRASIL. Ministério da Saúde. Manual técnico: pré-natal e puerpério atenção qualificada e humanizada. Brasília, DF: Ministério da Saúde, 2006. Disponível em: <http:// bvsms.saude.gov.br/bvs/publicacoes/manual_pre_natal_ puerperio_3ed.pdf $>$. Acesso em: 01 ab. 2015.

CARVALHO, P. I. et al. Fatores de risco para mortalidade neonatal em coorte hospitalar de nascidos vivos. Epidemiol. Serv. Saúde, v.16, n.3, p. 185-194, 2007.

FRANCESCHINI, D. T. B.; CUNHA, M. L. C. Associação da vitalidade do recém-nascido com o tipo de parto. Revista Gaúcha de Enfermagem, v.28, n.3, p. 324-330, 2007.

FREITAS, P. F.; SAVI, E. P. Desigualdades sociais nas complicações da cesariana: uma análise hierarquizada. Cad. Saúde Pública, v.27, n.10, p. 2009-20, 2011.

FISHER, N. A. B.; CARABALLO, M. G.; SÁNCHEZ, M. M. C. Factores epidemiológicos y Apgar bajo al nacer. Revista Cubana de Obstetricia y Ginecología, v. 37, n. 3, p. 320-329, 2011.

GAIVA, M. A. M.; FUJIMORI, E.; SATO, A. P. S. Mortalidade neonatal em crianças com baixo peso ao nascer. Rev Esc Enferm USP, v.48, n.5, p.778-786, 2014.

GONÇALVES, A. C.; COSTA, M. C. N.; BRAGA, J. U. Análise da distribuição espacial da mortalidade neonatal e de fatores associados, em Salvador, Bahia, Brasil, no período 2000-2006 . Cad. Saúde Pública, v. 27, n. 8, p.
1581-1592, 2011.

KASSAR, S. B. et al. Determinants of neonatal death with emphasis on health care during pregnancy, childbirth and reproductive history. J Pediatr., v.89, n.3, p.269-77, 2013.

KILSZTAJN, S. et al. Vitalidade do recém-nascido por tipo de parto no Estado de São Paulo, Brasil. Cadernos de Saúde Pública, v. 23, n. 8, p. 1886-1892, 2007.

KNUP, V. M. A. O.; MELO, E. C. P.; OLIVEIRA, R. B. Distribuição do parto vaginal e da cesariana no município do Rio de Janeiro no período de 2001 a 2004. Esc Anna Nery Rev Enferm., v. 12, n. 1, p. 39-44, 2008.

LANSKY et al. Pesquisa Nascer no Brasil: perfil da mortalidade neonatal e avaliação da assistência à gestante e ao recém-nascido. Cad. Saúde Pública, v. 30, p. S192-S207, 2014.

MAIA, L. T. S.; SOUZA, W. V.; MENDES, A. C. G. Diferenciais nos fatores de risco para a mortalidade infantil em cinco cidades brasileiras: um estudo de caso-controle com base no SIM e no SINASC. Cad. Saúde Pública, v. 28, n. 11, p. 2163-2176, 2012.

MIRANDA, F. J. S.; FERNANDES, R. A. Q. Assistência pré-natal: estudo de três indicadores. Rev. enferm. UERJ, v. 18, n. 2, p. 179-184, 2010.

MOUTA, R. J. O. et al. Relação entre posição adotada pela mulher no parto, Integridade perineal e vitalidade do recémnascido. Rev. enferm. UERJ, v. 16, n. 4, p. 472-476, 2008.

NASCIMENTO et al. Determinantes da mortalidade neonatal: estudo caso-controle em Fortaleza, Ceará, Brasil. Cad. Saúde Pública, v. 28, n. 3, p. 559-572, 2012.

OLIVEIRA et al. Escore de Apgar e mortalidade neonatal em um hospital localizado na zona sul do município de São Paulo. Einstein, v. 10, n. 1, p. 22-28, 2012.

ORGANIZAÇÃO MUNDIAL DE SAÚDE (OMS). Assistência ao parto normal: um guia prático. Genebra: 1996. 53p.

RIBEIRO, A. M. et al. Fatores de risco para mortalidade neonatal em crianças com baixo peso ao nascer. Rev Saúde Pública, v. 43, n. 2, p. 246-55, 2009.

ROCHA et al. Mortalidade neonatal e evitabilidade: uma análise do perfil epidemiológico. Rev. enferm. UERJ, v. 19, n. 1, p. 114-120, 2011.

SALVO, F.; HUGO et al. Factores de riesgo de test de Apgar bajo en recién nacidos. Rev. chil. pediatr. v. 78, n. 3, p. 253-260, 2007.

SOARES, E. S.; MENEZES, G. M. S. Fatores associados à mortalidade neonatal precoce: análise de situação no nível local*. Epidemiol. Serv. Saúde, v. 19, n. 1, p. 51-60, 2010. 
THEME FILHA, M. M. et al. Taxa de cesariana nos estabelecimentos públicos e privados: análise do Sistema de informações sobre nascidos vivos no município do Rio de Janeiro, 1996-2006. In: Brasil. Ministério da Saúde. O modelo de atenção obstétrica no setor de saúde suplementar no Brasil: cenários e perspectivas. Rio de Janeiro: ANS, 2008. p. 127-140. Disponível em: <http:// bvsms.saude.gov.br/bvs/publicacoes/modelo_atencao_ obstetrica.pdf $>$. Acesso em: Acesso em: 01 ab. 2015.

VIEGAS, D.; MORAES, R. V. Neonatologia para o estudante de pediatria e de enfermagem pediátrica. São Paulo: Atheneu, 1996. 760p.

Recebido: $10 / 12 / 2014$

Aceito: 08/06/2015 